\title{
El control interno basado en el modelo COSO
}

Santa Cruz Marín, Marinelly ${ }^{1}$

Universidad Peruana Unión (UPeU), Perü

Recibido 28 de julio de 2014 - Aceptado el 28 de octubre de 2014

\section{Resumen}

En la dinámica del entorno actual, se ha creado la necesidad de integrar conceptos en todos los niveles de las diversas áreas administrativas y operativas, con el fin de ser competitivos y responder a las nuevas exigencias empresariales, surge así un Treadway Commission (COSO) y sus componentes, siendo una estrategia para el desarrollo y eficiente uso de los recursos de una Treadway Commission (COSO) y sus componentes, siendo una estrategia para el desarrollo y eficiente uso de los recursos de una
organización. Por lo tanto, el modelo refleja el dinamismo en el ambiente de control, así como la evaluación de riesgos, no solo influyen en las actividades de control, sino que puede también poner de relieve la conveniencia de considerar el manejo de la información y la en las actividades de control, sino que puede tambien poner de relieve la conveniencia de considerar el manejo de la información y la
comunicación dentro de un proceso supervisado y corregido de acuerdo con las circunstancias. No se trata de un proceso en serie, en el que un componente incide exclusivamente sobre el siguiente, sino que es interactivo y multidireccional, en tanto cualquier componente puede influir, y de hecho lo hace, en cualquier otro.

Palabras clave: Control interno, modelo COSO, organización.

Abstrac

In the dynamics of the current environment, has created a need for integrating concepts at all levels of the various administrative and operational areas, in order to be competitive and respond to new business requirements, and a new concept of internal control arises where provides a common structure: the model Committee of Sponsoring Organizations of The Treadway Commission (COSO) and its components, being a strategy for the development and efficient use of resources in an organization. Therefore, the model reflects the dynamic control environment and risk assessment, not only influence control activities, but can also highlight the desirability of considering the management of information and communication in a process overseen and corrected according to the circumstances. There is a serial process, where one component affects only on the following, but is interactive and multidirectional, as any component can influence, and indeed it does in any other

Keywords: Internal control, Coso model, organization

A lo largo de los años el control interno ha sido definido desde diferentes perspectivas, pero que congruentemente sigue los mismos objetivos de lograr las metas empresariales, promover eficiencia operativa y la confiabilidad en la información financiera de las unidades económicas. En virtud a la diversidad de conceptos, definiciones e interpretaciones existentes en torno al control interno es publicado, en 1992, el modelo COSO en los Estados Unidos (Normas generales de control interno, 2 producto de pecerno $y$ representando un proceso efecturdo por el consejo de administración, la dirección y el resto del personal de la entidad, diseñado con el objetivo de proporcionar un grado de seguridad razonable en cuanto a la consecución de los objetivos dentro de las siguientes categorías: a) Eficacia y eficiencia de las operaciones; b) Fiabilidad de la información financiera; c) Cumplimiento de las leyes y normas aplicables (Mantilla B., 2000).

En este sentido, la importancia del modelo COSO radica en que puede ser usado como base para revisar la efectividad de los sistemas de control y determinar cómo mejorarlos (Alberto, 2004), presentando pautas de funcionamiento para prevenir la violación de la (Ricardo,

En la actualidad, según la Sociedad del Comercio Exterior, del total de firmas formales del Perú, las microempresas representan un $98.3 \%$, de las cuales las pequeñas empresas representan el $1.5 \%$ y las medianas y grandes el $0.5 \%$. Además, el gremio destacó que las mypes aportan el $17.8 \%$ de la producción nacional, y el $39 \%$ de ellas se ubican en Lima Metropolitana. Sin embargo, según la Sociedad Peruana de Pyme cada año surgen 12 mil mypes, pero solo el 10\% supera el ão de actividad.
Es por ello, que se estima que una de las causas por las cuales fracasan las mypes en el Perú, de acuerdo a lo publicado por el Instituto de Estadística e Informática (INEI), es que del $40 \%$ de las micros y pequeñas empresas que decidieron recibir algún tipo de capacitación para su negocio el 2011, solo el $14.2 \%$ de las mypes se capacitan en gestión empresarial.

\section{Control interno}

\section{Concepto}

El control interno puede ser abordado mediante dos criterios: como un plan y un proceso en una organización. Según Estipiñán (2006) el control interno es un plan de organización y el conjunto de métodos y procedimientos que sirven para salvaguardar los activos y mostrar una información contable fidedigna. De este modo, bajo el mismo criterio Santillana (2001) agrega que el control interno permite la eficiencia operativa y estimula la adhesión a las políticas prescritas por la administración. Conjuntamente, Perdomo (2004) asume que este plan es adoptado por empresas públicas, privasas o mixtas Por otro lado, el contros públicas, pivadas o mixtas. Por otro lado, el control interno se define como un proceso que permite el logro y la medición de los objetivos especificos de una entidad, mediante diversos componentes interrelacionados (Mantilla, 2005). A simismo, Mantilla y Cante (2005) señalan que el control interno es un procedimiento continuo que involucra a todos los niveles del personal.

\section{Objetivos decontrol interno}

El control interno tiene como principales objetivos: lograr las metas empresariales, promover eficiencia operativa y la confiabilidad en la información financiera de las unidades económicas. Respecto al primer objetivo, Santillana (2001) cita que el control interno permite Santillana (2001) cita que el control interno permite
fomentar el pleno respeto, apego, observancia y adhesión a las políticas establecidas por la administración de la entidad, con el fin de alcanzar los objetivos trazados. De este modo, el control interno garantiza el éxito de una entidad, afirmando la consecución de los objetivos básicos empresariales (Cooper \& Lybrand, 1997).

Por otro lado, Mantilla (2005) menciona que al realizar un control interno, dentro de una organización, se alcanzaría el uso un control intemo, dentro de una orgaizacion, se Asimismo, es de impontante identificar claramente las metas y estandares de operación para medir el desempeño y calificar la eficiencia, eficacia y economía; elementos indispensables para conseguir un desempeño integral de la empresa (Santillana G onzález, 2001).
Evidenciando que no realizan un adecuado proceso de control interno en sus actividades; pues cada una de ellas soluciona sus problemas y limitaciones de acuerdo a su capacidad y criterio, siendo este hecho un impedimento para lograr representatividad y alcanzar un crecimiento económico y social significativo.

Por otra parte, considerando el tercer objetivo, el último autor (2001) señala que control interno certifica la razonabilidad, confiabilidad, oportunidad e integridad de la información financiera y complementaria administrativa y operacional que se genera en la entidad, con el fin de que sirvan como base para la toma de decisiones para garantizar bienestar futuro. Además, Perdomo (2004) añade que el control interno permite localizar posibles errores administrativos, contables y financieros. A ñadiendo además, otros objetivos específicos como: proteger y salvaguardar los bienes, valores, propiedades y demás activos de la empresa; detectar desperdicios innecesarios de material, tiempo,

etcéter

\section{Enfoques decontrol interno}

\section{Enfoquetradicional decontrol intemo}

De acuerdo a Estupiñán (2006) el enfoque tradicional define al control interno como un plan de organización y el conjunto de métodos y procedimientos, que se rige bajo los siguientes elementos concretos:

- Organización: Un plan lógico y claro de las funciones organizacionales que establezca líneas claras de autoridad y responsabilidad.

- Sistemas y procedimientos: Un sistema adecuado para la autorización de transacciones y procedimientos seguros para registrar los resultados en términos financieros.

- Personal: el personal debe tener aptitud, capacitación y experiencia requerida para cumplir sus obligaciones satisfactoriamente.

- Supervisión: Las operaciones deben ser examinadas o procedimientos de autocontrol en las dependencias administrativas y de apoyo logístico.

Por otro lado, M antilla (2005) afirma que en la segunda generación enfoque tradicional, se desarrolló con mucha fuerza las oficinas, las jefaturas y los comités, así como los manuales y procedimientos de operación a través de roles como: el rol de ejecución y el rol de evaluación. 


\section{E nfoque contemporáneo de control interno}

I enfoque contemporáneo "M odelo coso" no define al control interno como un plan; por lo contrario lo presenta como un proceso, el cual es ejecutado por la junta directiva y por el resto del personal de una entidad. EI mismo que consta de cinco componentes interrelacionados, que se derivan de la forma cómo la administración maneja el ente, los cuales se clasifican como: ambiente de control, evaluación de riesgos, actividades de control, información y comunicación, supervisión y seguimiento (Estupiñán Gaitán, 2006)

De tal forma Mantilla (2005) expresa que actulmente De tal forma, Manilla (2005) expresa que actualmente el control interno centra esfuerzos en la calidad derivada del posicionamiento en los más altos niveles estratégicos y directivos, como requisito mínimo que garantiza la eficiencia.

Dentro de este contexto, las empresas ahora han modificado sus controles, esquematizando su evaluación y conclusiones más en el riesgo de control que en los procedimientos, sabiendo lograr una adecuada estructura de control interno de la organización (Estupiñán Gaitán \& Estupiñán Gaitán, 2006).

inalmente de acuerdo a lo antes mencionado, se afirma e el COSO es el punto de partida para importantes a de control de nuevos instrumentos financieros, gubernamental y sistemas de
información.

\section{M odelo de control interno coso}

Origen

La historia del modelo COSO está enmarcada a dos aspectos fundamentales: Contexto en la que se origina y el grupo de trabajo que hizo la propuesta. Como forma de solucionar la diversidad de conceptos, definiciones e
interpretaciones existentes en torno al control interno, es publicado en 1992, el modelo coso en los Estados publicado en 1992, el modelo coso en los Estados
Unidos (Normas generales de control Interno, 2007). A simismo, M elini (2005) el modelo coso nace como una respuesta a la preocupación generalizada respecto de los razonantes casos jurisprudenciales que evidencian situaciones límites, donde las quiebras están originadas en fallas de los administradores respecto al manejo de los patrimonios de las empresas.

De esta forma, los escándalos financieros ocurridos en Europa y, posteriormente en Norteamérica a inicios del siglo XXI, estremecieron el mundo empresarial y sirvieron para posicionar al coso como un marco adecuado para la evaluación del control interno de las compañías (Fonseca Luna, 2011)
Por otro lado, el grupo de trabajo estuvo constituido por representantes de la A merican Accounting A ssociation (AAA), American Institute of Certified Public Accountants (AICPA), Financial Executive Institute (FAI), Institute of Internal Auditors (IIA) e Institute of $M$ anagement $A$ ccountants (IMA), y sus siglas COSO que corresponde al Committee of Sponsoring Organizations de la Tread-way Commission (Cooper \& Lybrand, 1997). A simismo, Mantilla (2005) menciona el mismo concenso de cinco asociaciones profesionales de líderes, las cuales lo han convertido al COSO, en el estándar internacional de control interno.

\section{Concepto}

El modelo "COSO" puede ser definido como: técnicas de control interno y criterios para una eficiente gestión de la organización. Siendo así, el informe COSO plantea que el control interno sea un proceso integrado, que forme parte de los procesos de los negocios y no pesados mecanismos burocráticos añadidos a los mismos (Cooper \& Lybrand, 1997). Sin embargo, Diego (2011) alude que el modelo COSO es más que un conjunto de procedimientos de control, se trata más bien de un marco procedimientos de control, se tra más bien de un marco que considera la tanea de un control inteno de una empresa como un fenomeno complejo y dinámico. Por lo contrario, para Fonseca Luna (2011) el coso establece los criterios para lograr una gestión efectiva y eficiente en la evaluación del riesgo y en la evaluación de los controles internos en las organizaciones. A simismo, el presente modelo brinda un plan estratégico que incide esencialmente en los resultados de la gestión(Cuellar, 2009).

\section{Objetivos}

Los objetivos primordiales del modelo cOSO son: integrar diversos conceptos del control interno y ser la base para evaluar los sistemas de control interno en las entidades. Según, Cooper \& Lybrand (1997) el modelo COSO pretende que los interlocutores tengan una referencia conceptual común sobre el control interno para garantizar un buen gobierno corporativo. De esta manera, el modelo COSO fue creado con la finalidad de conceptualizar el control interno, principalmente para la emisión de información financiera (Lara, 2012).

Por otra parte, el modelo coso presenta pautas de funcionamiento para prevenir la violación de la estructura de organización y de sus sistemas (Ricardo, 2005). También Mantilla (2004) asevera que el modelo coso puede ser usado como base para revisar la efectividad de los sistemas de control y determinar cómo mejorarlos.

\section{Elementos del coso}

Ambiente de contro

El ambiente de control marca la base del funcionamiento de una empresa e influye en la concienciación de sus empleados respecto al control. Según Orellana (2002) el ambiente de control es la piedra angular de todos los demás componentes del control interno, aportando disciplina y estructura. A simismo, el ambiente de control da el tono de una organización, donde la administración asigna autoridad y responsabilidad (M antilla, 2005). En esencia, este componente es el principal elemento sobre el que se sustenta 0 actúan los otros cuatros componentes, siendo a su vez indispensable para la realización de los objetivos de control de una organización (Estupiñán Gaitán \& Estupiñán Gaitán, 2006).

Por otra parte, Betancur \& López (2007) mencionan que el ambiente de control constituye las pautas de comportamiento en una organización y las formas cómo comportamiento en una organizacion y las formas cómo control de la gente. De esta forma, lo trascendente de cualquier negocio es su gente, sus atributos individuales, incluyendo la integridad, los valores éticos y la competencia (N úñez Hurtado, 2012). También, M antilla (2004) refiere que los factores del ambiente de control incluyen la integridad, los valores éticos y la competencia de la gente de la entidad; el estilo de operación de la administración y cómo la administración organiza y desarrolla a su gente.

Evaluación de riesgos

a evaluación de riesgos radica en la identificación y el análisis de los riesgos relevantes de una organización. Para Orellana (2002) el mencionado elemento del informe coso consiste en la identificación, cuantificación y priorización de potenciales eventos que pudieran tener impacto y/o consecuencias significativas para la organización y la consecución de los objetivos fijados por la empresa Del mismo modo, Estipiñ 2006) refiere que la evaluación de riesgos se refiere a riesgos específicos asociados con los cambios.

Además, una condición previa a la evaluación de los resgos es el establecimiento de los objetivos en cada nivel de la organización y, en base a dicho análisis, determinar la forma en que los riesgos deben ser gestionados (Cooper \& Lybrand, 1997).

Es por ello que todas las entidades enfrentan diversos riesgos procedentes de fuentes tanto internas como externas, los cuales deber evaluarse a nivel de la entidad y actividad; y, a partir de ello, establecer mecanismos para identificar, analizar y tratar los riesgos. (V ega Fernández \& Pérez Díaz, 2010).

Actividades de control

Las actividades de control pueden estar agrupadas como: políticas y procedimientos que aseguren las directrices de la dirección. A sí para Betancur \& López (2007) las actividades de control se hacen visibles en las políticas organizacionales, las cuales deben estar desarrolladas para todos los niveles, ya sea estratégico, administrativo y operacional. Dicho de otro modo, es necesario un conjunto de reglas y normas de actuación, que rijan tanto para los procesos informativos contables como, para el operativo de la empresa (recursos financieros y humanos) lo que ayudaŕa tener un mejor control de los procesos (Núñez Hurtado, 2012).

Además de ello, Mantilla (2000) menciona algunos procedimientos de control como: revisiones de alto nivel, funciones directivas 0 actividades administrativas, procesamiento de información, controles físicos, indicadores de desempeño y segregación de responsabilidad. Por consiguiente, las actividades de control ayudan a asegurar que se lleven a cabo aquellas acciones identificadas como necesarias para afrontar los riesgos, y así conseguir los objetivos de la entidad (Gutiérrez Colque, 2012).

Información y comunicación

La información y comunicación se refieren básicamente a captar e intercambiar la información en una organización y el apoyo al cumplimiento de las responsabilidades. Cada entidad debe desarrollar sistemas interrelacionados que permitan al personal de la entidad capturar e intercambiar la información necesaria para conducir, administrar y controlar sus operaciones (Ricardo, 2005). De tal forma Mantilla (2005) hace referencia que los sistemas de información producen reportes, contienen información operacional, financiera y relacionada con el cumplimiento, para operar y controlar el negocio. 
Por otra parte, Veraz Rabines (2013) infiere que en esta actividad se refiere a la identificación, recopilación y comunicación de información de la organización, de modo tal que permita, en el tiempo y la forma, ser oportuna y contribuya a que los empleados cumplan con sus responsabilidades. En concordancia, es necesario que cada miembro de una organización conozca el rol que le corresponde desempeñar en su interior y que cuenten con información periódica y oportuna que requieren para orientar sus acciones. (Cervantes Corona, 2009).

\section{Monitoreo}

monitoreo deber realizarse a través de dos procedimientos como: actividades de revisión y evaluaciones que aseguren el buen funcionamiento de los controles. Conforme a ello, M antilla (2005) expone que el monitoreo incluye actividades regulares de administración y supervisión y otras acciones personales realizadas en el cumplimiento de sus obligaciones. gualmente, resulta necesaria la revisión de las actividades de control a través del tiempo, dado que toda organización tiene áreas en donde estos están en volución y neces arer reforzados 0 modificados (Cooper \& Lybrand, 1997).

Por otro lado, las evaluaciones pueden ser desarrolladas por distintas instancias, según la importancia de la actividad, magnitud de los riesgos, estructura organizativa y políticas internas de cada organización Orellana Flores, G aete A raya, \& G aete Becerra, 2002).

En definitiva, cada entidad debe monitorear el proceso total, y debe considerar como necesario hacer modificaciones. De esta manera, el sistema de control puede reaccionar dinámicamente, cambiando a medida que las condiciones lo justifiquen ( $N$ uñez Hurtado, 2012).

\section{Referencias}

Alberto, M. B. (2004). Control interno de los nuevos instrumentos financieros. B ogotá: Editorial Kimpres

Á lvarez, J. (1984). Burocracia y poder político en el régimen franquista. España: Instituto Nacional de Administración Pública.

\section{Condusiones}

El control interno constituye una parte fundamental en toda organización, ya que de este depende que todas las operaciones se realicen adecuadamente y que los riesgos exigentes puedan ser manejables.

EI modelo Coso es el resultado del trabajo de un comité representado por contadores públicos y auditores, así como administradores y financieros que, en algún momento, decidieron estandarizar una metodología para la evaluación del control interno

El modelo coso es un instrumento eficaz en la evpectos a ser consi control, ealuán de riesgos, actividades de información y comunicación, así como el monitoreo.

Si bien todas las organizaciones necesitan llevar a cabo prácticas de control, este informe está especialmente orientado a aquellas en las que por su envergadura, requieren y están en condiciones de aplicar mecanismos formales y preestablecidos de control para evitar 0 reducir los fraudes, riesgos y conductas inadecuadas que puedan surgir, tanto por parte del personal, como por clientes y proveedores.

Al implementar las prácticas sugeridas en el modelo COSO, las organizaciones consiguen controlar más eficiente, eficaz y transparentemente su operatoria. De tal modo, que puedan parametrizar y formalizar las técnicas de medición, el control resulta simple y efectivo. En síntesis, las prácticas de este modelo son una herramienta altamente recomendable en materia de control interno para las organizaciones.

Álvarez, J. (2009). Estilos de liderazgo en la policia local de la comunidad valenciana. Valencia: Universidad de $V$ alencia.

Álves, J. (2000). Liderazgo y clima organizacional. Revista de Psicología del D eporte, IX(1-2), 123 133.
A rico, J., \& Tula, J. (2005). Diccionario de política (Catorceava ed.). M éxico: Siglo XXI Editores.

Baca, L., Bokser, J., Castañeda, F., Cisneros, H., \& Pérez, G. (2000). Léxico de la Política. M éxico: FLACSO M éxico Publicaciones.

Ballesteros, V. (diciembre de 2010). I mportancia de la organización en los procesos de la gestion pública. IAPEM (77), 245-247.

Bastiani, J., Ruiz, L., Estrada, E., Cruz, T., \& A paricio, J. (2012). Perfiles Educativos. Politica Indigena Educativa, 34(135), 8-25.

Batesi, R. (1989). Práctica critica de la administración educativa. España: Universidad de V alencia.

Beltrán, M . (1997). La formacion y los empleados publicos. (u. a. madrid, Ed.) REIS, 51-68.

Betancur, H. D., \& López, J. E. (2007). A proximación conceptual y metodológica de la administración de riesgos, una nueva forma de entender el contro Interno y de administrar las PYMES del eje cafetero. Pereira: Universidad Tecnológica de Pereira.

Betancur, H. D., \& López, J. E. (2007). A proximación Conceptual y M etodológica de la Administración de Riesgos, Una Nueva F orma de Entender el Control Interno y de Administrar Las PYMES del Eje Cafetero. Pereira: Universidad Tecnológica de Pereira.

Blanco, M. (setiembre de 1994). Hacia una antropologia de la burocracia. Nueva Antropología, XIV (046), 117-129.

Cervantes Corona, G. (2009). M odelo de Atención al Cliente en el Área de Siniestro-Autos en el Ramos Asegurador. M éxico: Insitituto Politécnico Nacional.

Cervantes, O., \& Corrales, G. (1987). Administración en instituciones públicas: antologia (V ol. I). San J osé: Universidad Estatal a Distancia.

Chiavenato, I. (1989). Introducción a la teoría genera de la administración. Santa Fe, México: M cG rawHill.
Cooper \& Lybrand. (1997). Los nuevos conceptos de control interno. M adrid: Ediciones Díaz de Santos S.A.

Cornejo, M . (agosto de 2007). Liderazgo de excelencia. Veritas, 51(1632), 48.

Cuellar, G. A . (2009). Informe Ejecutivo del C OSO Universidad del Cauca.

Da Silva, R. (2002). Teoría de la Administración. M éxico: Thomson.

Daft, R. (2006). La experiencia del liderazgo (Tercera ed.). M èxico: Thomson

Daft, R. (2007). Teoría y diseño organizacional (N ovena ed.). Santa Fe, M éxico: Cengage L earning Editores.

Dianine, A . (2010). Perfil del líder: hacia un liderazgo virtuoso. España: Editorial formatic M y C.

Diego, C. (2011). Contra el Fraude. A rgentina: Editoria Granica.

Estrada, S. (diciembre de 2006). M odelos de liderazgo en organizaciones cambiantes. Scientia Et Technica, XII (032), 295-300.

Estrada, S. (mayo de 2007). Liderazgo a través de la historia. Scientia Et Technica, XIII (034), 343-348.

Estupiñán G aitán , R., \& Estupiñán G aitán, O. (2006) Análisis financiero y de gestión. Bogotá: Ecoe Ediciones.

Estupiñán Gaitán, R. (2006). Control interno y fraudes. Bogotá: ECO Ediciones.

Faria, J., \& M eneghetti, F. (2011). Burocracia como organização, poder e controle. (portuguese). Revista de Administração de Empresas, LI (5), 424-439.

Fernández, J. (2002). Liderazgo empresarial en tiempos de cambio, según Sun Tsu. Harvard D eusto Business Review, XXIII(109), 64-71.

Fernández, S. (julio de 2004). Sobre el concepto y el estudio del liderazgo político. Psicología Política, 15(29), 7-29. 
Fonseca Luna, O. (2011). Sistemas de control interno para las organizaciones. Lima: Insituto de Investigación de A ccountability y Control.

García, M . (2002). Habilidades sociales del líder político. Universidad Complutense de M adrid. M adrid: Editorial de la Universidad M adrid.

Gutiérrez Colque, R. (2012). Propuesta de una guía como herramienta de control interno para la unidad de auditoría interna en la detección de fraudes para las empresas industriales. Bolivia Universidad M ayor de San Andrés.

Heady, F. (2000). Administración pública: una perspectiva comparada. M éxico: Fondo de cultura económica.

Hell riegel, D., J ackson, S., \& Slocum, J. (2006). Administración: un enfoque basado en competencias. México: Thomson.

Hermida, J., \& Serra, R. (1989). Administración y estrategia. B uenos A ires, A rgentina: Ediciones Machi.

Huaylupo, J. (2007). El liderazgo: un poder relativo. Revista de Ciencias Sociales, I(15), 103-124.

Ibañez, E. (2004). Liderazgo y compromiso social (Primera ed.). M éxico: Universidad A utónoma de San Luis de Potosí.

ara, A. (2012). Toma el control de tu negocio. México: LID Editorial Empresarial.

Lucero, A . (junio-julio de 2002). B urocracia municipal y el proyecto educativo. Revista del Centro de Investigacion. Universidad la Salle, 5(018-017), $17-28$.

Mantilla B ., S. A. (2000). Control interno estructura conceptual integrada. Colombia: Ecoe Ediciones.

M antilla B., S. A., \& Cante S., S. Y. (2005). Auditoria del control interno. Bogotá: Ecoe Ediciones.

M antilla, S. A. (2005). Control interno (Informe C OSO). Bogotá: Ecoe Ediciones.
M aqueda, J., \& Llaguno, J. (1995). M arketing estratégico para empresas de servicios. España Editorial Dias de Santos.

M arín, D. (julio-diciembre de 2006). El sujeto humano en la administración: una mirada crítica. Cuadernos de Administración, XIX(032), 135-156.

M orgade, G. (mayo - agosto de 2007). B urocracia Educativa, T rabajo D ocente y G énero: supervisoras que conducen "poniendo el cuerpo". Educación y Sociedad, 28(99), 400 - 425

M uñoz, W. (diciembre de 2007). El servicio civil: una necesidad para una admnistración postburocrática. Revista de Economía y Administración, 69(69), 39 56.

M urillo, F. (2006). Una direccion escolar para el cambio: del liderazgo transformacional al liderazgo distribuido. Revista Electrónica Iberoamericana sobre calidad, eficacia y cambio en educacion. $4(4), 13-14$.

N ormas Generales de Control Interno. (2007). Auditorí Interna de la Nación.

Normas Generales de Control Interno. (2007). Auditoría Interna de la Nación, 44.

Núñez Hurtado, G. (2012). Evaluación a la gestión administrativa y control interno en los procesos de recaudación de regalias del sistema municipal de estacionamiento rotativo tarifado Simert y su incidencia presupuestaria en el período comprendido de junio 2010 a junio 2011. A mbato Ecuador: Universidad Técnica de A mbato.

Orellana Flores, L., Gaete A raya, J., \& G aete B ecerra, H. (2002). Alternativas de valorizacion del sistema de control interno en las empresas. Chile: UCHile.

Palomo, M. (2010). Liderazgo y motivación de equpos de trabajo (Sexta edición ed.). M adrid, España: ESIC Editorial.

Patiño, C. (2005). El origen del poder de 0 ccidente Estado, guerra y orden internacional. Madrid: Taurus.
Perdomo M oreno, A . (2004). Fundamentos de control interno. M éxico: Cengage.

Pérez, G. (setiembre de 2012). Gestión, liderazgo y valores en la administración de centros educativos. "G estión de liderazgo y val ores en el Centro Educativo Escuela Fiscal M ixta N 208 Ciudad de A zogues, de la ciudad de G uayaquil, durante el periodo lectivo 2011 - 2012". tesis, UTPL, Guayaquil.

Pérez, V. (octubre de 2002). La lucha contra Ia corrupción en Venezuela : balance y perspectiva en el marco de la nueva administración pública. VII Congreso Internacional del CLAD sobre la Reforma del Estado y de la A dministración Pública. Lisboa, Portugal: Universidad de los A ndes ( $V$ enezuela).

Ramírez, L. (2007). Teoría administrativa. Documento de docente, Universidad Santiago de Chile, Departamento de Gestión y Políticas Públicas.

Restrepo, M . (enero-junio de 2011). La administración pública en la Constitución de 1991: sincretismo involuntario entre la burocracia, el gerencialismo la gobernanza. Revista Estudios Socio-jurídicos, XIII (001), 365-388.

Ribeiro, C. (2011). Perfil de liderazgo de los gerentes de banco hacer A gencia de brasil en el estado de mapa. Monografia, Universidad de Brasil, brasil.

Ricardo, M. (2005). Enfoques de la auditoría de estados contables. A rgentina: Universidad Nacional de Litoral.

Ricardo, M . (2005). Enfoques de la auditoría de estados contables. A rgentina: Universidad $\mathrm{N}$ acional de Litoral.

Rosas, H., T revera, A., \& Rosas, M . (Enero.junio de 2009). L a estructura burocrática y su flexibilidad para la innovación en los modelos de M ax W eber Robert M erton. Revista Internacional La Nueva Gestión Organizacional, 4(8), 98-111.

Sánchez, J. (2001). M oral pública en los procesos de buen gobierno. M éxico: Plaza y V aldez Editores.

Santa Cruz, A . (1997). Comparando sistemas burocráticos: los casos de Estados U nidos, J apón y México. Espiral, VII(008), 93-110.

Santillana González, J . R. (2001). Establecimientos de sistemas de control interno. México: Ediciones Paraninfo.

Torres, Z. (2002). Teoría general de la administración M éxico: Editorial Patria.

Vásquez, R. (diciembre de 2006). W eber y su concepción de la democracia posible. Andamios, III(5), 213-236.

V ega Fernández , L., \& Pérez Díaz, F. (2010). M etodología aplicada al control interno para los estudiantes de la carrera de Contabilidad de cuarto año. O bservatorio de la Economía Latinoamericana, 133.

Vélez, A . (2007). Los clásicos de la gerencia. Bogotá, Colombia: Editorial Universidad del Rosario.

Veras Rabinez, R. T. (2013). Implementación de políticas y procedimientos de control interno en empresas del Sistema Financiero en estado de liquidación. Lima: Universidad Nacional de San $\mathrm{M}$ arcos.

V on Sprecher, R. (2005). Teorías sociológicas: Introducción a los clásicos (Tercera ed.). Córdova: Editorial Brujas.

Zabludovsky, G. (2007). Sociología y cambio conceptual. M éxico: Siglo XXI Editores 